\title{
Blood amyloid $\beta$ oligomerization as a biomarker of Alzheimer's disease: a blinded validation study
}

\section{Young Chul Youn}

Chung-Ang University Hospital https://orcid.org/0000-0002-2742-1759

\section{Byoung Sub Lee}

PeopleBio Inc.

\section{Gwang Je Kim}

PeopleBio Inc.

\section{Ji Sun Ryu}

PeopleBio Inc.

Kuntaek Lim

PeopleBio Inc.

\section{Ryan Lee}

PeopleBio Inc.

Jeewon Suh

Seoul National University Bundang Hospital

\section{Young Ho Park}

Seoul National University Bundang Hospital

Jung-Min Pyun

Seoul National University Bundang Hospital

Nayoung Ryu

Seoul National University Bundang Hospital

Min Ju Kang

Veterans Health Service Medical Center

\section{Hye Ryoun Kim}

Chung-Ang University College of Medicine

\section{Sungmin Kang}

PeopleBio Inc.

\section{Seong Soo A. An}

Gachon University

SangYun Kim ( $\nabla$ neuroksy@snu.ac.kr)

https://orcid.org/0000-0002-9101-5704

\section{Research}


Keywords: Oligomer, Amyloid ß, Alzheimer's disease, Biomarker, Multimer Detection System, Blood

Posted Date: January 3rd, 2020

DOl: https://doi.org/10.21203/rs.2.19960/v1

License: (c) (1) This work is licensed under a Creative Commons Attribution 4.0 International License. Read Full License 


\section{Abstract}

INTRODUCTION: Oligomeric amyloid $ß(A ß)$ is one of the major contributors to the pathomechanism of $A D ; A ß$ oligomerization in plasma can be measured using a Multimer Detection System-Oligomeric $A ß$ (MDS-OAß) after incubation with spiked synthetic Aß. METHODS: We evaluated the clinical sensitivity and specificity of the MDS-OAß values by inBlood TM OAß test using heparin-treated plasma samples from $52 \mathrm{AD}$ patients in comparison with 52 community-based subjects with normal cognition (NC). The inclusion criterion was proposed by the NINCDS-ADRDA and additionally required for the least 6 months of follow-up from the initial clinical diagnosis in the course of AD. RESULTS: The MDS-OA $\beta$ values were $1.43 \pm 0.30 \mathrm{ng} / \mathrm{ml}$ in $A D$ and $0.45 \pm 0.19(p<0.001)$ in NC, respectively. Using a cut-off value of 0.78 $\mathrm{ng} / \mathrm{ml}$, the results revealed that $100 \%$ sensitivity $92.31 \%$ specificity. DISCUSSION: MDS-OAß to measure plasma $A \beta$ oligomerization is a valuable blood-based biomarker for clinical diagnosis of $A D$, with high sensitivity and specificity.

\section{Introduction}

Studies investigating the development of a biomarker for the early diagnosis of Alzheimer's disease (AD) have increased throughout medical communities worldwide. Many studies have suggested that amyloid $\beta(A \beta)$ oligomers could present as potent toxic species compared to insoluble fibrils (1-4). A $\beta$ oligomers, playing a key-role in $A D$ pathogenesis, have become a promising candidate for $A D$ diagnosis. Efforts to measure $A \beta$ oligomers in humoral fluids are currently underway (5-7). However, cost-effective and noninvasive diagnostic methods to detect $A \beta$ oligomers are not currently available in clinical practice. Furthermore, a clinically applicable method to measure $A \beta$ oligomers in the blood has not yet been reported. Due to the invasiveness of lumbar puncture, the accessibility of patients for cerebrospinal fluid (CSF) analysis is low, limiting its general usage. Therefore, blood analysis would be a beneficial complement to this shortcoming. However, there are several limitations of using plasma $A \beta$ as bloodbased biomarker of $A D$. Firstly, $A \beta$ is highly diluted in blood $(8,9)$. Furthermore, $A \beta$ can bind to and interfere with other protein and peptides $(10,11)$, and undergo degradation (12) and self-aggregation (13) in the blood.

An atypical approach, called the Multimer Detection System-Oligomeric A $(M D S-O A \beta)$, was introduced to measure the oligomerization dynamics in plasma samples after spiking synthetic $A \beta$ (14). This essentially utilizes the MDS technique, which can selectively detect oligomers in a given sample $(15,16)$. One study found that the level of $A \beta$ oligomers increased after spiking $A \beta$ and incubation, in plasma samples of $A D$ patients but not in healthy normal subjects (14). The elevated levels of $A \beta$ oligomers closely correlated with conventional AD biomarkers, such as CSF AB42 and Pittsburgh compound B (PIB) positron emission tomography (PET) standard uptake ratio, CSF phosphorylated tau, and CSF total tau (17). In this study, we aimed to validate the accuracy of MDS-OA $\beta$ for measuring $A \beta$ oligomerization dynamics in heparin-treated plasma samples from patients with $A D$ and healthy controls. The objective of this study was to assess the sensitivity and specificity of the MDS-OA $\beta$ test in differentiating plasma from AD patient and subjects with normal cognition (NC). The positive predictive value (PPV) and 
negative predictive value (NPV) were also evaluated. In addition, the MDS-OA levels in AD patients was compared to the Clinical Dementia Rating (CDR) scores, a numeric scale used to quantify the severity of dementia symptoms.

\section{Methods}

The study was supported by a contract research organization (CRO). This clinical study was approved by the Korea Ministry of Food and Drug Safety (MFDS) and performed in accordance with its approved protocol (No.753). Approval by the institutional review board (IRB) of Seoul National University Bundang Hospital (IRB no.: E-1703/386-001) and Chung-Ang University Hospital (IRB no.:1722-008-272) were obtained. Furthermore, the study was conducted in compliance with Good Clinical Practice established by the Korea Ministry of Food and Drug Safety and International Council for Harmonization of Technical Requirements for Pharmaceuticals for Human Use and ethical principles of the Declaration of Helsinki.

\section{Materials}

The samples were selected from repositories. The inclusion criteria for the NC group were as follows: (1) a community-based population; (2) no abnormality on the Health Screening Questionnaire (18); (3) absence of memory complaints; (4) a Korean Dementia Screening Questionnaire score $\leq 6$ (19); (5) normal general cognition (within 1 standard deviation of the age- and education-adjusted norms of the Korean version of the Mini-Mental State Examination (20) and a score > 26); (6) intact activities of daily living (K-IADL $\leq$ 0.42); (7) no depression (the short-form Geriatric Depression Scale $\leq 7$ ); (8) no history of thyroid dysfunction, vitamin B12 deficiency, or folate deficiency; (9) No abnormalities on the MRI scan. A more stringent inclusion criteria was applied to patients with AD dementia (ADD): (1) the probable AD criteria proposed by the National Institute of Neurological and Communicative Disorders and Stroke and the Alzheimer's Disease and Related Disorders Association (NINCDS-ADRDA) (21), as well as the DSM-IV; (2) a follow-up of at least 6 months to determine the clinical course of AD by experienced neurologists; (3) male or female patients between the ages of 50 to 80, (4) education for at least 6 years. The case records were reviewed thoroughly. Exclusion criteria included the presence of cognitive impairment other than $A D$, stroke, and delirium. Detailed demographic data of subjects with NC and AD are presented in Table 1. 
Table 1

Demographics of the subjects included in the study

\begin{tabular}{|lllll|}
\hline Group & CDR & No. of subjects & Age (years) & MMSE \\
\hline NC & 0 & 52 & $60.5 \pm 7.4$ & N/A \\
\hline ADD & 0.5 & 25 & $71.3 \pm 9.0$ & $19.4 \pm 4.1$ \\
& 1 & 17 & $72.3 \pm 10.1$ & $17.9 \pm 6.8$ \\
& 2 & 9 & $68.2 \pm 7.9$ & $20.2 \pm 6.3$ \\
& 3 & 1 & 73 & 20 \\
\hline
\end{tabular}

Data presented as mean \pm standard deviation.

Abbreviations: NC, community-based normal cognition; ADD, Alzheimer's disease dementia; MMSE, Mini-Mental State Examination.

The sample size was calculated considering the sensitivity and specificity for the clinical diagnosis of AD (22) and clinical trials of amyloid PET (23). The sensitivity of $70.9 \%$ for diagnosing AD by Beach et al. was set as the predetermined sensitivity $\left(P_{0}\right)(22)$ and the sensitivity of $88.0 \%$ based on clinical trials of Vizamy ${ }^{\mathrm{TM}}$ Flutemetamol F $18(23)$ as the expected sensitivity $\left(\mathrm{P}_{1}\right)$. Based on the sensitivities following the hypothesis and equation by K. Hajian-Tilaki (24), a sample size of 47 was calculated. Initially, 60 samples for each group were extracted from the repositories. Of these, the CRO excluded 16 samples due to medical history and sample quality issues. Finally, heparin-treated plasma samples from 52 patients with $A D$ and 52 subjects with NC were selected considering the $10 \%$ dropout for this study (Figure. 1). The MDS-OA $\beta$ measurements using the inBlood ${ }^{\mathrm{TM}}$ OAß test (PeopleBio Inc., Gyeonggi-do, Republic of Korea) were taken twice successively, and the average was used. The exclusion criteria of samples were as follows: if an error occurred twice (error in test and re-test); if there was only one error that was not corrected in the additional measurement; if the number of remaining samples to be re-tested was insufficient. All 104 samples were randomized and anonymized for the test. For each sample, a random assignment number generated by an independent statistician from Seoul National University Bundang Hospital IRB was assigned as the sample identification number.

\section{Assay description and procedure}

The inBloodTM ${ }^{T M}$ OAß test (PeopleBio Inc., Gyeonggi-do, Republic of Korea) was utilized to quantify MDS$O A \beta$ values in heparin-treated plasma from patients with $A D$ and NC. The inBlood ${ }^{T M} O A B$ test, based on MDS was a modified atypical sandwich immunoassay for measuring oligomeric A $\beta$. In the MDS method, the epitope-overlapping antibodies specific for the $\mathrm{N}$-terminus of $A \beta$ were used to capture and to detect the $A \beta$ oligomers. The epitopes for the $6 \mathrm{E} 10$ and W0-2-HRP antibodies overlapped at the N-terminus of $A \beta$, and mouse monoclonal anti-6E10 (BioLegend, San Diego, CA, USA) and anti-W0-2-HRP antibodies (Absolute Antibody Ltd, Oxford, UK) were therefore used to capture and to detect $A \beta$ oligomers, respectively. 
Prior to the assay, aliquots of plasma samples were thawed at $37^{\circ} \mathrm{C}$ for 15 minutes. As indicated in the assay protocol of the inBloodTM OAß test, PBR-1 (synthetic $A \beta$ made by PeopleBio Inc.) was spiked into plasma and the mixture was incubated at $37^{\circ} \mathrm{C}$ for $48 \mathrm{hr}$. The incubated plasma sample mixture and serially diluted standard samples were added to each well of the plates. The plates were incubated at about 20 to 25 degrees Celsius for 1 hour. After washing three times with washing buffer, W02-HRP antibody was added to the wells, and the plates were incubated for 1 hour at about 20 to 25 degrees Celsius. To increase the sensitivity of detection, $100 \mu \mathrm{l} /$ well of enhanced chemiluminescence substrate solution (Rockland Immunochemicals Inc., Limerick, PA, USA) was added, and the Relative Luminescence Unit (RLU) signal was detected using a Victor 3TM multi-spectrophotometer. Dilutions providing signal in the linear range of the standard curves were used for the conversion to RLU values to determine the concentration of oligomerized $A \beta$.

\section{Statistical analysis}

Average MDS-OA $\beta$ value from the inBlood ${ }^{\mathrm{TM}} \mathrm{OA}$ tests of each group were compared using a two independent sample t-test. Receiver operating characteristic (ROC) analysis of the MDS-OA $\beta$ values of the plasma samples from patients with $A D$ and subjects with NC was performed to obtain the corresponding cut-off value for the highest area under the curve (AUC). The cut-off value obtained by the ROC analysis was reverified by calculating the reference interval. The reference interval and the associated Confidence Interval $(\mathrm{Cl})$ were determined by a non-parametric method according to the guideline of Clinical and Laboratory Standards Institute (CLSI). This was determined to be positive if the individual MDS-OA value was higher than the cut-off value. In addition to the calculation of the sensitivity and the specificity to differentiate between plasma samples of patients with $A D$ and subjects with $N C$, the positive and negative predictive values were also calculated. Furthermore, to confirm the correlative trend of MDS-OA $\beta$ values and AD severity, MDS-OA $\beta$ levels were compared with the CDR scores. Statistical significance $p$ value was set at 0.05 .

\section{Results}

None of the 104 samples were excluded during the test procedure. The average MDS-OA $\beta$ values of the $A D$ and $N C$ samples were $1.43 \pm 0.30 \mathrm{ng} / \mathrm{ml}$ and $0.45 \pm 0.19 \mathrm{ng} / \mathrm{ml}$, respectively, and there was a significant difference between the two groups $(p<0.001)$ (Fig. 2). The ROC analysis indicated an optimal cut-off value $(0.78 \mathrm{ng} / \mathrm{ml})$, which allowed the best differential discrimination between patients with $A D$ and NC subjects. In order to closely verify the optimal cut-off value, we induced the reference interval by biding by the guideline from CLSI C28-A3; 'the (indirect) techniques are perhaps more appropriately employed using data from individuals who are relatively healthy.' The $95 \%$ reference interval subjected to $\mathrm{NC}$ is $0.783 \mathrm{ng} / \mathrm{ml}$ and therefore, is approximate to the cut-off value of ROC analysis. The AUC was 0.999. As a result, all 52 samples of the AD group were found to be positive, and 48 samples of the NC group were negative, indicating $100 \%$ sensitivity (95\% Cl: $100 \%$ ) and $92.31 \%$ specificity (95\% Cl: 85.07 99.55\%). The PPV and NPV were $92.86 \%$ and $100 \%$, respectively (Table 2 ). 
Table 2

Characteristics of MDS-OA $\beta$ between subjects with Alzheimer's disease and normal cognition when using a cut-off value of $0.78 \mathrm{ng} / \mathrm{ml}$

\begin{tabular}{|lllll|}
\hline & ADD & NC & Total & P-value $^{*}$ \\
\hline Positive & 52 & 4 & 56 & \\
\hline Negative & 0 & 48 & 48 & $<0.0001$ \\
\hline Total & 52 & 52 & 104 & 0.0006 \\
\hline $\begin{array}{l}\text { Sensitivity (\%) } \\
(95 \% \text { Cl) }\end{array}$ & 100 & & & \\
\hline $\begin{array}{l}\text { Specificity (\%) } \\
(95 \% \text { Cl) }\end{array}$ & $(100)$ & 92.31 & $(85.07-99.55)$ & \\
\hline Abbreviations: NC, community-based normal control; ADD, Alzheimer's disease dementia & \\
\hline * Ratio difference as to whether the sensitivity is greater than 70.9\%; Z-test & \\
\hline
\end{tabular}

The MDS-OA $\beta$ levels were significantly higher in patients with a CDR score of $0.5(1.46 \pm 0.33 \mathrm{ng} / \mathrm{ml}, \mathrm{n}=$ 25), $1(1.53 \pm 0.21, n=17)$, and $2(1.26 \pm 0.21, n=9)$, compared with CDR $0(0.45 \pm 0.19, n=25)(p<0.001)$ (Fig. 3). Interestingly, the average of MDS-OA levels decreased as the CDR score in AD patients increased; however, there was no significance $(p>0.05)$.

\section{Discussion}

Many researchers have chosen $A B$ oligomers as a biomarker for the diagnosis of $A D$ as it satisfies the criteria of an ideal biomarker, which was proposed by the Ronald and Nancy Reagan Research InstituteNIA in 1998 (25). Several studies have substantiated the positive correlation between the levels of $A \beta$ oligomers in plasma and the likelihood of $A D(26)$, and found the sensitivity and specificity to be less than $85 \%$ (6). The MDS-OA $\beta$ measures oligomerization dynamics of $A \beta$ in the blood without the need for specialized equipment, unlike conventional techniques which directly measure $A \beta$ molecules using larger machines due to the low concentration of target molecules in the blood (14). In this study, the oligomerization tendencies of $A \beta$ in $A D$ and normal plasma were measured using the MDS-OA , and we demonstrated that the sensitivity and specificity were $100 \%$ and $92.3 \%$, respectively; therefore MDS-OA $\beta$ has very high sensitivity and specificity in distinguishing $A D$ from NC. We used a stringent patient recruitment criterion for this study. For example, AD patients were followed-up for at least 6 months by experienced neurologists to rule out the possibility of cognitive impairment caused by any other disease, and community-based NC subjects without cognitive decline, were enrolled.

Although the data was not shown in this study, while assessing 29 cases of the AD, all patients showed high MDS-OAß levels, and a PIB or Florbetaben PET was conducted. The standardized uptake value radio 
(SUVR) of 25 cases were positive, and 3 cases had a positive visual rating but negative SUVR. One case had a negative amyloid PET but had a typical CSF profile of AD. This patient may have a soluble form AB, which failed to produce $A \beta$ plaques in the brain; therefore, only showing changes in the CSF biomarker $(27-30)$. While the MDS-OA $\beta$ measures dynamics of $A \beta$ oligomerization (14), amyloid PET only reveals the fibrillar form of $A \beta$ in the brain (31), which may have caused the discrepancy. The other possible reason is that changes in CSF biomarkers may have occurred before the amyloid PET change. A $\beta$ oligomerization tendency in plasma may reflect as early as changes in the CSF biomarker, and signify AD regardless of the solubility of $A \beta$.

Many studies have argued that biomarkers, such as brain volume and CSF A 442, p-tau, and t-tau which indicates the downstream effects of $A D$, show an increase in severity as the disease progress and formed a graph of the sigmoid curve $(32,33)$. However, the average of MDS-OA 3 level in this study was the highest at CDR score 0.5 and the lower as AD progress (Fig. 3). MDS-OA $\beta$ measures the oligomerization dynamics of $A \beta$, which corresponds to the derivative of the sigmoid function of $A \beta$ accumulation. It is possible that this biomarker changes during the early phase of $A D$, as shown with other biomarkers associated with processes upstream of the $A D$ pathomechanism, and decreases in expression as the disease progresses (34-36). Another possible explanation could be that the concentrations of neuronal injury/death biomarkers decrease after symptom onset, which suggests slowing of the acute neurodegenerative processes with symptomatic disease progression (37).

One limitation of the present study was the age difference between the AD and NC groups. However, we found that there was no correlation between MDS-OA $\beta$ levels and age in the $52 \mathrm{NC}$ subjects (range 5177 , mean $60.5 \pm 7.4$ ), and speculated that age difference was not a significant variable in influencing MDS-OA $\beta$ levels. Second, the MMSE score of AD increased in patients with CDR 2 (Table 1), but this is thought to be due to the patients being of a younger age than those with CDR 1 .

\section{Conclusions}

Plasma samples of $A D$ and NC subjects were differentiated using MDS-OA $\beta$, which measured the $A ß$ oligomerization tendency of plasma. Furthermore, MDS-OA $\beta$ was found to have high sensitivity and specificity. Based on the current findings, measuring the $A \beta$ oligomerization tendency in plasma could be a simple and reliable blood-based biomarker for the diagnosis of AD.

\section{Declarations}

\section{Acknowledgements}

Not applicable

\section{Funding}

This work was supported by the Korea Health Industry Development Institute [HI17C1864, 2017]. 


\section{Data availability of data and materials}

Data are available upon reasonable request.

\section{Authors' contributions}

SYK: conceptualization and design of the study and collection of the data. YCY: collection and analysis of the data, drafting and revision of the manuscript, and prepared all the figures. JSR, HRK, MJK and KL: collection and analysis of the data, and revision of the manuscript. BSL, GJK, RL, JS, YHP, J-MP, NR, SK and SSAA: revision of the manuscript.

\section{Ethics approval and consent to participate}

This research was approved by the Ministry of Food and Drug Safety and performed in accordance with its approved protocol (No.753). Approval by the institutional review board (IRB) of Seoul National University Bundang Hospital (IRB no.: E-1703/386-001) and Chung-Ang University Hospital (IRB no.:1722008-272) were obtained.

\section{Consent for publication}

All authors consented to publication of this paper

\section{Competing interests}

All authors have no conflicts of interest to declare.

\section{References}

1. Tomic JL, Pensalfini A, Head E, Glabe CG. Soluble fibrillar oligomer levels are elevated in Alzheimer's disease brain and correlate with cognitive dysfunction. Neurobiol Dis. 2009;35(3):352-8.

2. Um JW, Nygaard HB, Heiss JK, Kostylev MA, Stagi M, Vortmeyer A, et al. Alzheimer amyloid-beta oligomer bound to postsynaptic prion protein activates Fyn to impair neurons. Nat Neurosci. 2012;15(9):1227-35.

3. Izzo NJ, Staniszewski A, To L, Fa M, Teich AF, Saeed F, et al. Alzheimer's therapeutics targeting amyloid beta 1-42 oligomers I: Abeta 42 oligomer binding to specific neuronal receptors is displaced by drug candidates that improve cognitive deficits. PLoS One. 2014;9(11):e111898.

4. Cline EN, Bicca MA, Viola KL, Klein WL. The Amyloid-beta Oligomer Hypothesis: Beginning of the Third Decade. J Alzheimers Dis. 2018;64(s1):S567-S610.

5. Sun L, Zhong Y, Gui J, Wang X, Zhuang X, Weng J. A hydrogel biosensor for high selective and sensitive detection of amyloid-beta oligomers. Int J Nanomedicine. 2018;13:843-56.

6. Zhang J, Peng M, Jia J. Plasma amyloid-beta oligomers and soluble tumor necrosis factor receptors as potential biomarkers of AD. Curr Alzheimer Res. 2014;11(4):325-31. 
7. Kuhbach K, Hulsemann M, Herrmann Y, Kravchenko K, Kulawik A, Linnartz C, et al. Application of an Amyloid Beta Oligomer Standard in the sFIDA Assay. Front Neurosci. 2016;10:8.

8. Toledo JB, Vanderstichele H, Figurski M, Aisen PS, Petersen RC, Weiner MW, et al. Factors affecting Abeta plasma levels and their utility as biomarkers in ADNI. Acta Neuropathol. 2011;122(4):401-13.

9. Mehta PD, Pirttila T, Patrick BA, Barshatzky M, Mehta SP. Amyloid beta protein 1-40 and 1-42 levels in matched cerebrospinal fluid and plasma from patients with Alzheimer disease. Neurosci Lett. 2001;304(1-2):102-6.

10. Kuo YM, Kokjohn TA, Kalback W, Luehrs D, Galasko DR, Chevallier N, et al. Amyloid-beta peptides interact with plasma proteins and erythrocytes: implications for their quantitation in plasma. Biochem Biophys Res Commun. 2000;268(3):750-6.

11. Kuo YM, Emmerling MR, Lampert HC, Hempelman SR, Kokjohn TA, Woods AS, et al. High levels of circulating Abeta42 are sequestered by plasma proteins in Alzheimer's disease. Biochem Biophys Res Commun. 1999;257(3):787-91.

12. Wang J, Gu BJ, Masters CL, Wang YJ. A systemic view of Alzheimer disease - insights from amyloidbeta metabolism beyond the brain. Nat Rev Neurol. 2017;13(11):703.

13. Serem WK, Bett CK, Ngunjiri JN, Garno JC. Studies of the growth, evolution, and self-aggregation of beta-amyloid fibrils using tapping-mode atomic force microscopy. Microsc Res Tech. 2011;74(7):699708.

14. An SSA, Lee BS, Yu JS, Lim K, Kim GJ, Lee R, et al. Dynamic changes of oligomeric amyloid beta levels in plasma induced by spiked synthetic Abeta42. Alzheimers Res Ther. 2017;9(1):86.

15. An SS, Lim KT, Oh HJ, Lee BS, Zukic E, Ju YR, et al. Differentiating blood samples from scrapie infected and non-infected hamsters by detecting disease-associated prion proteins using Multimer Detection System. Biochem Biophys Res Commun. 2010;392(4):505-9.

16. Lim K, Kim SY, Lee B, Segarra C, Kang S, Ju Y, et al. Magnetic microparticle-based multimer detection system for the detection of prion oligomers in sheep. Int J Nanomedicine. 2015;10(Spec Iss):241-50.

17. Wang MJ, Yi S, Han JY, Park SY, Jang JW, Chun IK, et al. Oligomeric forms of amyloid-beta protein in plasma as a potential blood-based biomarker for Alzheimer's disease. Alzheimers Res Ther. 2017;9(1):98.

18. Christensen KJ, Moye J, Armson RR, Kern TM. Health screening and random recruitment for cognitive aging research. Psychol Aging. 1992;7(2):204-8.

19. Youn YC, Choi SH, Shin HW, Kim KW, Jang JW, Jung JJ, et al. Detection of cognitive impairment using a machine-learning algorithm. Neuropsychiatr Dis Treat. 2018;14:2939-45.

20. Han C, Jo SA, Jo I, Kim E, Park MH, Kang Y. An adaptation of the Korean mini-mental state examination (K-MMSE) in elderly Koreans: demographic influence and population-based norms (the AGE study). Arch Gerontol Geriatr. 2008;47(3):302-10.

21. Dubois B, Feldman HH, Jacova C, Dekosky ST, Barberger-Gateau P, Cummings $J$, et al. Research criteria for the diagnosis of Alzheimer's disease: revising the NINCDS-ADRDA criteria. Lancet Neurol. 2007;6(8):734-46. 
22. Beach TG, Monsell SE, Phillips LE, Kukull W. Accuracy of the clinical diagnosis of Alzheimer disease at National Institute on Aging Alzheimer Disease Centers, 2005-2010. J Neuropathol Exp Neurol. 2012;71(4):266-73.

23. Curtis C, Gamez JE, Singh U, Sadowsky CH, Villena T, Sabbagh MN, et al. Phase 3 trial of flutemetamol labeled with radioactive fluorine 18 imaging and neuritic plaque density. JAMA Neurol. 2015;72(3):287-94.

24. Hajian-Tilaki K. Sample size estimation in diagnostic test studies of biomedical informatics. J Biomed Inform. 2014;48:193-204.

25. Consensus report of the Working Group on: "Molecular and Biochemical Markers of Alzheimer's Disease". The Ronald and Nancy Reagan Research Institute of the Alzheimer's Association and the National Institute on Aging Working Group. Neurobiol Aging. 1998;19(2):109-16.

26. Zhou L, Chan KH, Chu LW, Kwan JS, Song YQ, Chen LH, et al. Plasma amyloid-beta oligomers level is a biomarker for Alzheimer's disease diagnosis. Biochem Biophys Res Commun. 2012;423(4):697702.

27. Price KA, Varghese M, Sowa A, Yuk F, Brautigam H, Ehrlich ME, et al. Altered synaptic structure in the hippocampus in a mouse model of Alzheimer's disease with soluble amyloid-beta oligomers and no plaque pathology. Mol Neurodegener. 2014;9:41.

28. Teller JK, Russo C, DeBusk LM, Angelini G, Zaccheo D, Dagna-Bricarelli F, et al. Presence of soluble amyloid beta-peptide precedes amyloid plaque formation in Down's syndrome. Nat Med. 1996;2(1):93-5.

29. Scholl M, Wall A, Thordardottir S, Ferreira D, Bogdanovic N, Langstrom B, et al. Low PiB PET retention in presence of pathologic CSF biomarkers in Arctic APP mutation carriers. Neurology. 2012;79(3):229-36.

30. Cairns NJ, Ikonomovic MD, Benzinger T, Storandt M, Fagan AM, Shah AR, et al. Absence of Pittsburgh compound $\mathrm{B}$ detection of cerebral amyloid beta in a patient with clinical, cognitive, and cerebrospinal fluid markers of Alzheimer disease: a case report. Arch Neurol. 2009;66(12):1557-62.

31. Rinne JO, Brooks DJ, Rossor MN, Fox NC, Bullock R, Klunk WE, et al. 11C-PiB PET assessment of change in fibrillar amyloid-beta load in patients with Alzheimer's disease treated with bapineuzumab: a phase 2, double-blind, placebo-controlled, ascending-dose study. Lancet Neurol. 2010;9(4):363-72.

32. Bateman RJ, Xiong C, Benzinger TL, Fagan AM, Goate A, Fox NC, et al. Clinical and biomarker changes in dominantly inherited Alzheimer's disease. N Engl J Med. 2012;367(9):795-804.

33. Jack CR, Jr., Knopman DS, Jagust WJ, Petersen RC, Weiner MW, Aisen PS, et al. Tracking pathophysiological processes in Alzheimer's disease: an updated hypothetical model of dynamic biomarkers. Lancet Neurol. 2013;12(2):207-16.

34. Youn YC, Park KW, Han SH, Kim S. Urine neural thread protein measurements in Alzheimer disease. J Am Med Dir Assoc. 2011;12(5):372-6.

35. Schuitemaker A, Dik MG, Veerhuis R, Scheltens P, Schoonenboom NS, Hack CE, et al. Inflammatory markers in $A D$ and $\mathrm{MCl}$ patients with different biomarker profiles. Neurobiol Aging. 
2009;30(11):1885-9.

36. Verwey NA, Schuitemaker A, van der Flier WM, Mulder SD, Mulder C, Hack CE, et al. Serum amyloid $p$ component as a biomarker in mild cognitive impairment and Alzheimer's disease. Dement Geriatr Cogn Disord. 2008;26(6):522-7.

37. Fagan AM, Xiong C, Jasielec MS, Bateman RJ, Goate AM, Benzinger TL, et al. Longitudinal change in CSF biomarkers in autosomal-dominant Alzheimer's disease. Sci Transl Med. 2014;6(226):226ra30.

\section{Figures}

\section{Samples selected from Repositories \\ $\operatorname{ADD}(n=60) \& N C(n=60)$}

\section{Exclusion of ADD (n=8) \& NC (n=8) samples by Contract Research Organization \\ - Poor sample quality \\ - Uncertain medical history}

\begin{tabular}{|c|}
\hline \multicolumn{1}{|c|}{ Eligible samples } \\
$\operatorname{ADD}(n=52)$ \& NC $(n=52)$
\end{tabular}

Figure 1

Enrollment of eligible subjects for MDS-OAß validation. ADD, Alzheimer's disease dementia; NC, community-based normal cognition. 


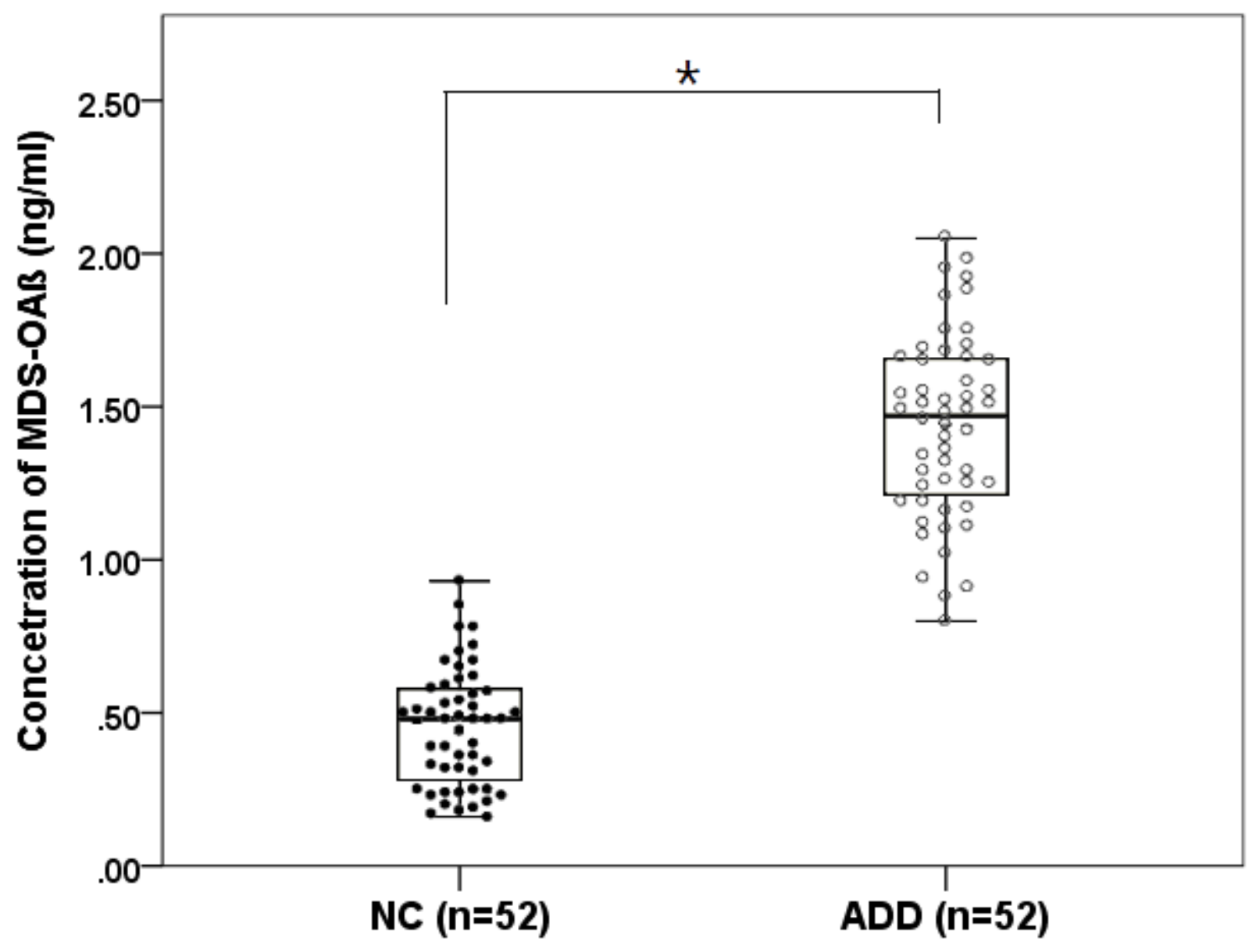

Figure 2

MDS-OA $\beta$ levels in heparin-treated plasma samples of Alzheimer's disease dementia patients and subjects with community-based normal cognition. The concentration of MDS-OA $\beta$ was significantly higher in ADD patients than in NC subjects. *t-test, $p<0.001 ; A D D$, Alzheimer's disease dementia; NC, community-based normal cognition. 


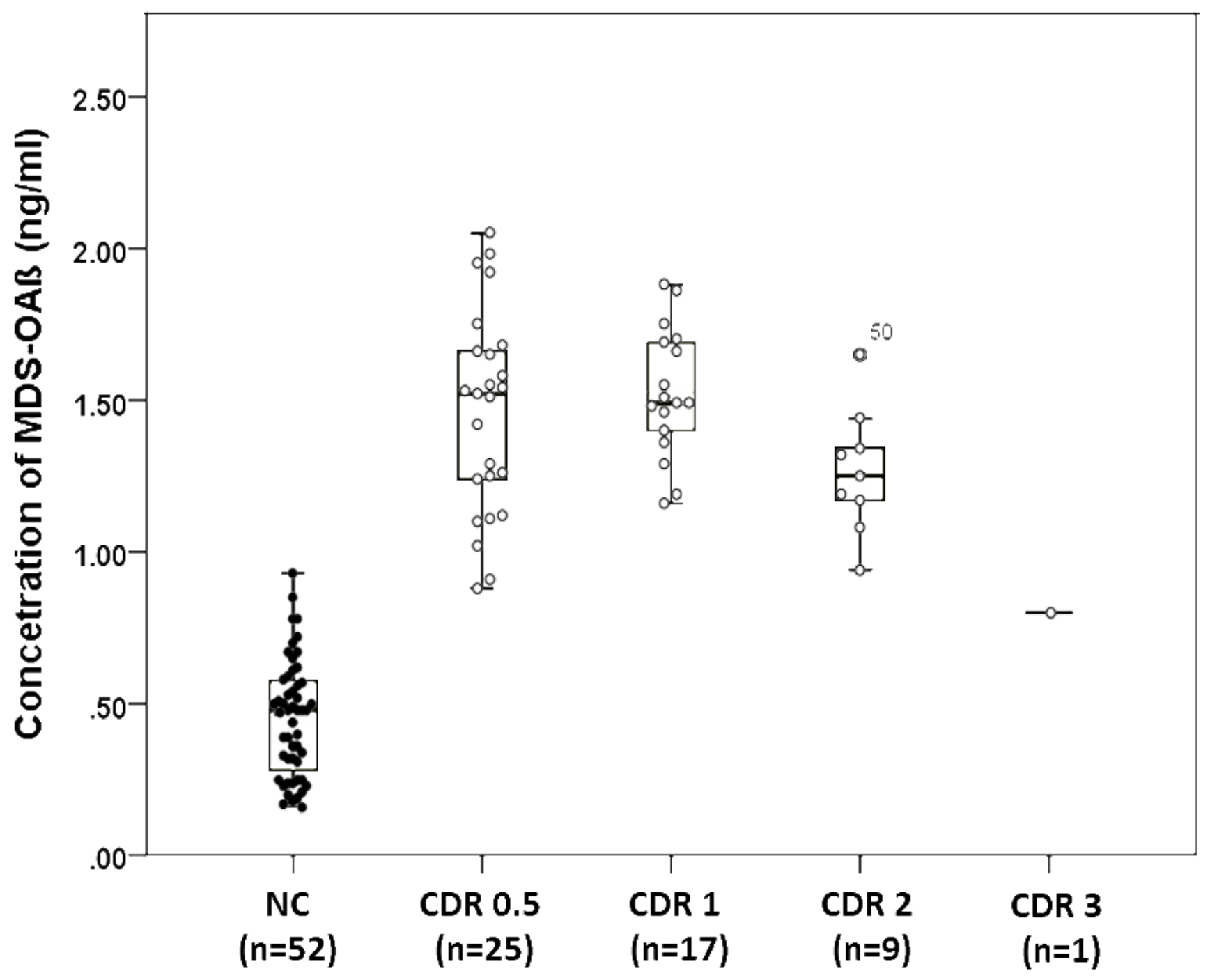

Figure 3

MDS-OAß level based on the clinical dementia rating. The decrease of the MDS-OAß mean with an increase of CDR score was not significant ( $p>0.05$ ); however, the MDS-OA $\beta$ levels were significantly higher in patients with a CDR score of $0.5,1$, and 2 versus those with a CDR score 0 was significant $(p<0.001)$. 